\title{
On the formation of tilted flux ropes in the Earth's magnetotail observed with ARTEMIS
}

\author{
S. A. Kiehas, ${ }^{1,2}$ V. Angelopoulos, ${ }^{1}$ A. Runov, ${ }^{1}$ M. B. Moldwin, ${ }^{3}$ and C. Möstl ${ }^{4,5}$
}

Received 15 November 2011; revised 6 April 2012; accepted 8 April 2012; published 26 May 2012.

[1] On 21 October 2010, ARTEMIS spacecraft P2, located at about $-57 R_{E_{G S M}}$ in the Earth's magnetotail, observed a series of flux ropes during the course of a moderate substorm. Subsequently, ARTEMIS spacecraft P1, located about $20 R_{E}$ farther downtail and farther into the lobe than P2, observed a series of TCRs, consistent with the flux ropes observed by $\mathrm{P} 2$. The dual-spacecraft configuration allows simultaneous examination of these phenomena, which are interpreted as an O-line, followed by a series of flux ropes/TCRs. An inter-spacecraft time of flight analysis, assuming tailward propagation of cross-tail aligned ropes, suggests propagation speeds of up to $\sim 2000 \mathrm{~km} / \mathrm{s}$. A principal axis investigation, however, indicates that the flux ropes were tilted between $41^{\circ}$ and $45^{\circ}$ in the GSM $x$-y-plane with respect to the noon-midnight meridional plane. Taking this into account, the tailward propagation speed of the different flux ropes is determined to be between 900 and $1400 \mathrm{~km} / \mathrm{s}$. The same timing analysis also reveals that the flux rope velocity increased progressively from one flux rope to the next. A clear correlation between the magnetic field and plasma flow components inside the flux ropes was observed. As possible mechanisms leading to the formation of tilted flux ropes we suggest (a) a progressive spreading of the reconnection line along the east-west direction, leading to a boomerang-like shape and (b) a tilting of flux ropes during their formation by non-uniform reconnection with open field lines at the ends of the flux ropes. The progressive increase in the propagation velocity from the first to the last flux rope may be evidence of impulsive reconnection: initially deep inside the plasma sheet the reconnection rate is slow but as reconnection proceeds at the plasma sheet boundary and possibly lobes, the reconnection rate increases.

Citation: Kiehas, S. A., V. Angelopoulos, A. Runov, M. B. Moldwin, and C. Möstl (2012), On the formation of tilted flux ropes in the Earth's magnetotail observed with ARTEMIS, J. Geophys. Res., 117, A05231, doi:10.1029/2011JA017377.

\section{Introduction}

[2] During magnetotail reconnection magnetic field lines from opposite sides of the current sheet are reconnected and transported toward the Earth and into the tail. In this classical two-dimensional near-Earth neutral line (NENL) model [Hones, 1976], the newly reconnected field lines form closed loop structures between the NENL and the distant X-line. These two-dimensional magnetic field line loops, referred to as plasmoids, propagate downtail [Schindler, 1974; Hones, 1977]. When there is a $B_{y}$-component in the magnetotail,

\footnotetext{
${ }^{1}$ Department of Earth and Space Sciences, University of California, Los Angeles, California, USA.

${ }^{2}$ Space Research Institute, Austrian Academy of Sciences, Graz, Austria.

${ }^{3}$ Department of Atmospheric, Oceanic, and Space Sciences, University of Michigan, Ann Arbor, Michigan, USA.

${ }^{4}$ Space Science Laboratory, University of California, Berkeley, California, USA.

${ }^{5}$ Institute of Physics, University of Graz, Graz, Austria.

Corresponding author: S. A. Kiehas, Department of Earth and Space Sciences, University of California, 595 Charles Young Dr. E., Box 951567, Los Angeles, CA 90095-1567, USA. (stefan.kiehas@gmail.com)

Copyright 2012 by the American Geophysical Union. 0148-0227/12/2011JA017377
}

the reconnected field lines are expected to create a threedimensional structure, identified as a flux rope [Hughes and Sibeck, 1987]. Hence, plasmoids can be seen as idealized two-dimensional simplifications of flux ropes [Moldwin and Hughes, 1991]. Flux ropes are characterized by a helical magnetic field topology. In the center of a flux rope, the magnetic field is purely axial and typically most intense, forming a core field. As one moves outwards from the center, the axial magnetic field weakens and the azimuthal magnetic field increases. Typical observations of flux ropes in the magnetotail include high speed tailward flows [e.g., Moldwin and Hughes, 1992a], and disturbances in the magnetic field due to its helical structure. For a cross-tail aligned flux rope, observations show a bipolar $B_{z}$ variation, decrease in $B_{x}$ and an increase in $B_{y}$, indicating the strong core field. Observed magnetic field signatures depend on the spacecraft trajectory through the structure [Moldwin and Hughes, 1991], however. Flux ropes resemble bulge-like plasma sheet disturbances the signatures of which are observed as traveling compression regions (TCRs) [Slavin et al., 1984] in the lobe. Lobe magnetic field lines drape around the flux rope and are compressed along the vertical direction to the current sheet. A spacecraft, located too far away from the flux rope to encounter the structure itself, can still detect a flux rope 

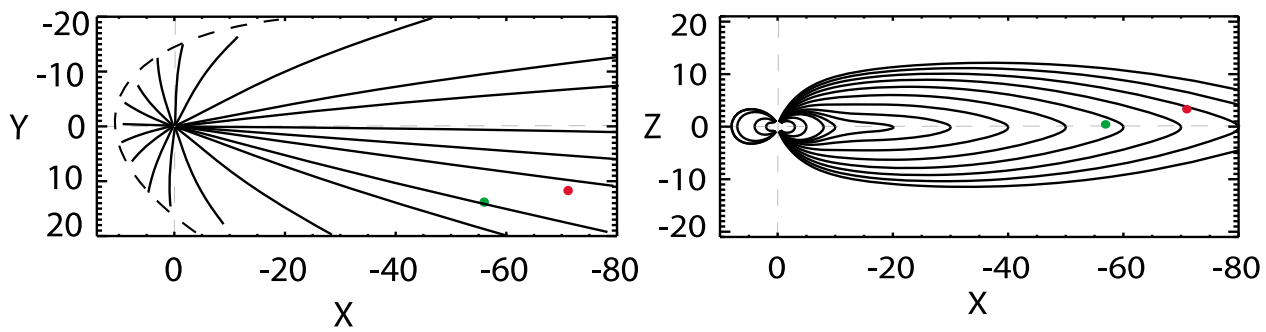

Figure 1. Position of the ARTEMIS spacecraft P1 (red) and P2 (green) on 21 October 2010 at 17:00 UT in the (left) $\mathrm{x}-\mathrm{y}$-plane and (right) $\mathrm{x}-\mathrm{z}$-plane in GSM. The field line configuration is derived from a T89 model.

passage via remote sensing of its lobe disturbances if it encounters the TCR [Slavin et al., 2003]. Typical TCR observations are characterized by the absence of plasma bulk flow and typical bipolar disturbances in $B_{z}$, accompanied by an enhancement in $B_{x}$ and $B_{\text {total }}$, resembling lobe magnetic field line compression [e.g., Slavin et al., 1993]. Plasmoids/ flux ropes and TCRs have been studied in the mid- and distant tail beyond $-20 R_{E}$ by IMP-8 [e.g., Moldwin and Hughes, 1992b; Taguchi et al., 1997], ISEE-3 [e.g., Hones et al., 1984; Baker et al., 1987; Richardson et al., 1987; Slavin et al., 1989; Moldwin and Hughes, 1992a], Galileo [Kivelson et al., 1993; Khurana et al., 1995] and Geotail spacecraft [Nagai et al., 1994, 1998; Mukai et al., 1996, 1998; Ieda et al., 1998, 2001; Slavin et al., 1998, 1999, 2002; Machida et al., 2000]. So far, only few multi-satellite observations of flux ropes and TCRs have been reported [Moldwin and Hughes, 1992b; Slavin et al., 1998, 1999]. With the new ARTEMIS mission [Angelopoulos, 2011; Sibeck et al., 2011], which is part of the extended THEMIS mission, we have the chance to get high-resolution data of dual spacecraft tail observations between about $-50 R_{E}>$ $X_{G S M}>-70 R_{E}$

\section{Instrumentation}

[3] The data used in this study were acquired from observations of the two ARTEMIS probes, which correspond to THB (P1) and THC (P2) of the THEMIS mission [Angelopoulos, 2008] and carry identical instrumentation. In this study we present magnetic field and plasma data collected by two instruments from about 17:00 UT to 18:00 UT on 21 October 2010. Magnetic field data were provided by the Fluxgate Magnetometer (FGM) [Auster et al., 2008] via DC magnetic field measurements with a temporal resolution of one vector per three seconds (spin resolution) in the survey mode available during the time of interest for this study. The ion velocities shown in this paper were derived from distribution functions provided by the Electrostatic Analyzer (ESA) [McFadden et al., 2008] in the $5 \mathrm{eV}$ to $25 \mathrm{keV}$ energy range with a temporal resolution of one 3-D distribution function per spin.

\section{Overview of the Observations}

[4] Between $\sim 17: 00$ UT and 18:00 UT on 21 October 2010, the ARTEMIS spacecraft P1 and P2 were located in the pre-midnight sector downtail at about $X_{G S M}=-71 R_{E}$ and $X_{G S M}=-57 R_{E}$, respectively (see Figure 1). They remained in the northern half of the tail during the entire interval between $Z_{G S M}=3.5$ and $4 R_{E}(\mathrm{P} 1)$ and $Z_{G S M}=0.5$ and $1.1 R_{E}(\mathrm{P} 2)$. According to the particle fluxes, $\mathrm{P} 1$ and $\mathrm{P} 2$ were located in the lobe regions at the beginning of the time of interest. Figures 2 and 3 show observations of P2 and $\mathrm{P} 1$, respectively, from 17:00 UT to 18:00 UT. We identify five events (labeled 1 to 5 chronologically) observed by both spacecraft. From $\sim 17: 12$ UT to 17:25 UT, P2 observed enhancements in the electron and ion particle fluxes, density, plasma pressure and temperature. Magnetic field disturbances were accompanied by a flow reversal in $v_{x}$. About two minutes after the flow reversal at P2, P1 observed a tailward traveling TCR (Event 1), inferred from the northto-south turning in $B_{z}$, accompanied by variations in $v_{z}$. This strong TCR $(\triangle B / B \sim 35 \%)$ was followed by a series of four smaller TCRs (Events 2 to 5 ) between $\sim 17: 30$ UT and 17:45 UT, showing enhancement in the total magnetic field strength, $B_{x}$, bipolar variations in $B_{z}$, and disturbances in $B_{y}, v_{y}$ and $v_{z}$. The magnetic field strength compression, mainly due to an enhancement in $B_{x}$, is reflected in magnetic pressure enhancements for each of the TCRs. Slightly before each of the four small-scale TCRs observed by P1, P2 observed disturbances in the magnetic field and plasma data. Events 2, 3 and 5, observed by P2 at $~ 17: 30$ UT, 17:35:30 UT and 17:43 UT respectively, were characterized by a decrease in the total magnetic field strength and $B_{x}$, bipolar signatures in $B_{z}$ and enhanced $B_{y}$. Furthermore, enhancements in the electron and ion particle fluxes, temperature, plasma pressure and ion beta were observed. The ion velocity showed tailward flows between $\sim 200$ to $400 \mathrm{~km} / \mathrm{s}$, accompanied by signatures in $v_{z}$ and $v_{y}$.

[5] Event 4, observed by P2 at $\sim 17: 40$ UT, exhibits significantly different signatures from Events 2, 3 and 5 and is characterized by an enhancement in the total magnetic field strength and $B_{x}$ as well as bipolar $B_{z}$ and $v_{z}$ signatures. The particle fluxes showed only a small enhancement of low energy particles. Temperature and plasma pressure remained at the background level. Due to the lack of significant particle fluxes and the compression in the total magnetic field strength, P2 did not encounter the plasma sheet flow region during Event 4, but instead observed the associated tailward propagating TCR.

\section{Passage of an O-Line (Event 1)}

[6] A comparison of magnetic field and plasma velocity observations by P1 and P2 between 17:10 UT and 17:30 UT is shown in Figure 4. Event 1, observed by $\mathrm{P} 2$ between about 17:12 UT and 17:25 UT, can be split into three distinct time intervals that are shown by different color bars in Figure 5. 


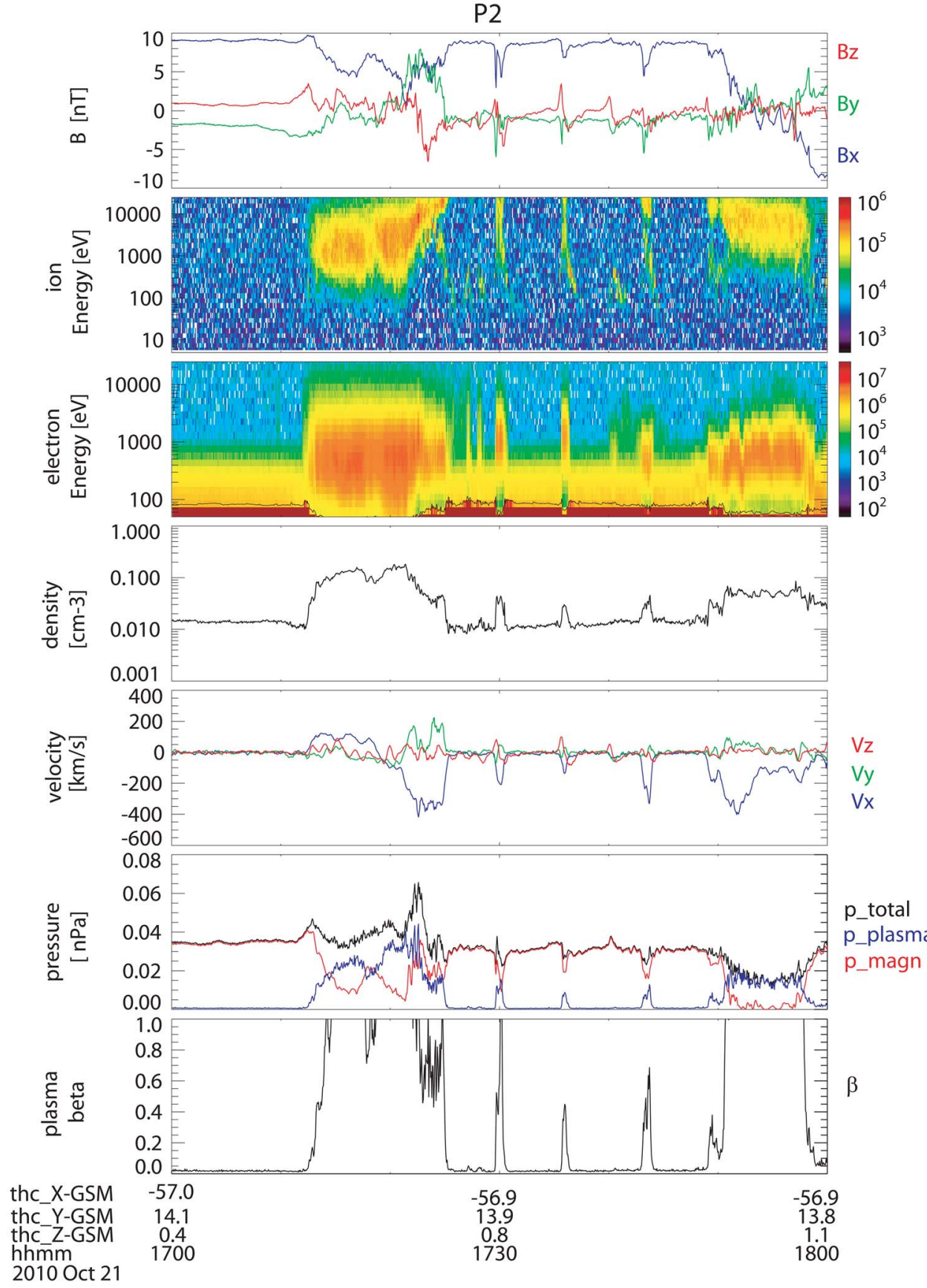

Figure 2. Overview of P2 observations on 21 October 2010 between 17:00 and 18:00 UT. From top to bottom: magnetic field $B$, omnidirectional ion energy spectrogram, omnidirectional electron energy spectrogram, plasma density, ion velocity, pressure, and plasma beta.

The first interval from about 17:12 UT to 17:19 UT is characterized by Earthward flow of $\sim 120 \mathrm{~km} / \mathrm{s}$, a decrease in $B_{x}$ and $B_{y}$ and positive $B_{z}$. The Earthward flow is predominantly parallel to the magnetic field. At 17:19 UT, the plasma flow changes from Earthward to tailward. We define the second interval as from 17:19 UT to 17:21 UT. During this time period, slow tailward flow of $\sim 100 \mathrm{~km} / \mathrm{s}$, accompanied by mainly positive $B_{z}$ was observed. The flow exhibited a stronger component normal to the magnetic field than during the first interval. At the beginning of the third interval, lasting from about 17:21 UT to 17:25 UT, the slow tailward flow turned into fast flow of $\sim 350 \mathrm{~km} / \mathrm{s}$, with a dominant component normal to the magnetic field. During this interval, $B_{z}$ turned from positive into negative and a strong $y$-component of the magnetic field was observed. These magnetic field and plasma characteristics during the last interval are typical of flux ropes. We interpret Event 1 as a flux rope, embedded in between the near-Earth neutral line (NENL) and the distant neutral line. This configuration of two connected reconnection lines is sometimes referred to as an "O-line" [e.g., Hoshino et al., 1996], since it naturally forms an O-type neutral line in between the two reconnection sites in a two- 


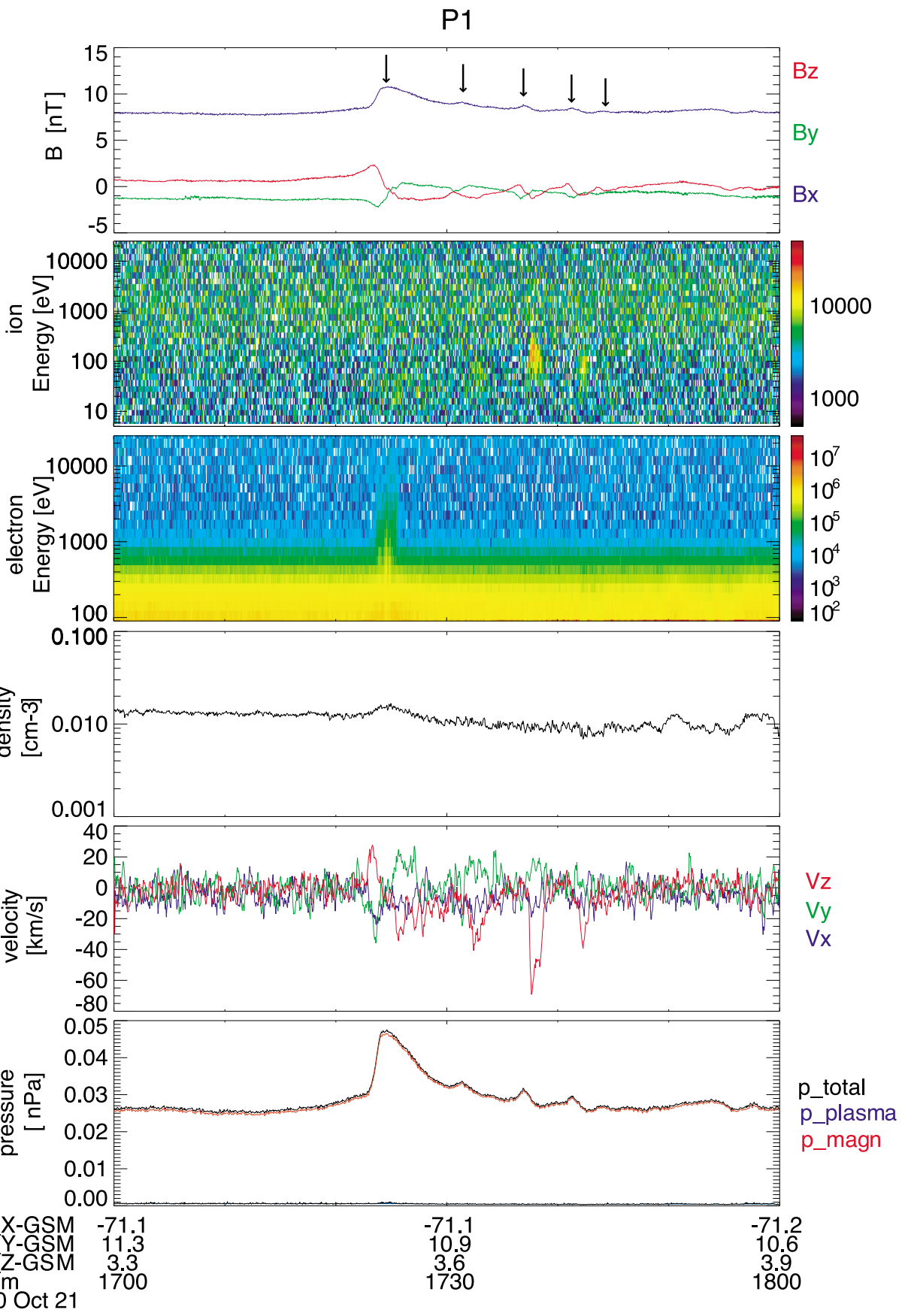

Figure 3. Overview of P1 observations on 21 October 2010 between 17:00 and 18:00 UT. From top to bottom: magnetic field $B$, omnidirectional ion energy spectrogram, omnidirectional electron energy spectrogram, plasma density, ion velocity, pressure, and plasma beta. The vertical black arrows in the first panel indicate the five TCRs.

dimensional configuration. The flow reversal at $\sim 17: 19$ UT serves as the main argument for the passage of an O-line. We interpret the Earthward flow during the first interval as reconnection outflow from the distant neutral line and the tailward flow during the following two intervals as reconnection jets from a near-Earth neutral line. Figure 6 shows a proposed scenario with the three panels referring to the three time intervals in Figure 5. During the first interval (first interval in Figure 5, corresponding to the blue horizontal bar in Figure 6a) of slow Earthward and mainly parallel plasma flow and positive $B_{z}, \mathrm{P} 2$ was located ahead of the flux rope and observed the reconnection outflow from the distant reconnection line. As the structure moved downtail, P2 engulfed the flux rope and observed slow tailward flow and positive $B_{z}$ (second interval in Figure 5, corresponding to the orange horizontal bar in Figure 6b). Consistent with previous observations from Geotail [Nishida et al., 1994], we interpret this slow flow as stagnant plasma that gets pushed forward as the reconnection outflow from the NENL moves downtail. This is confirmed by a slight density increase during the slow 


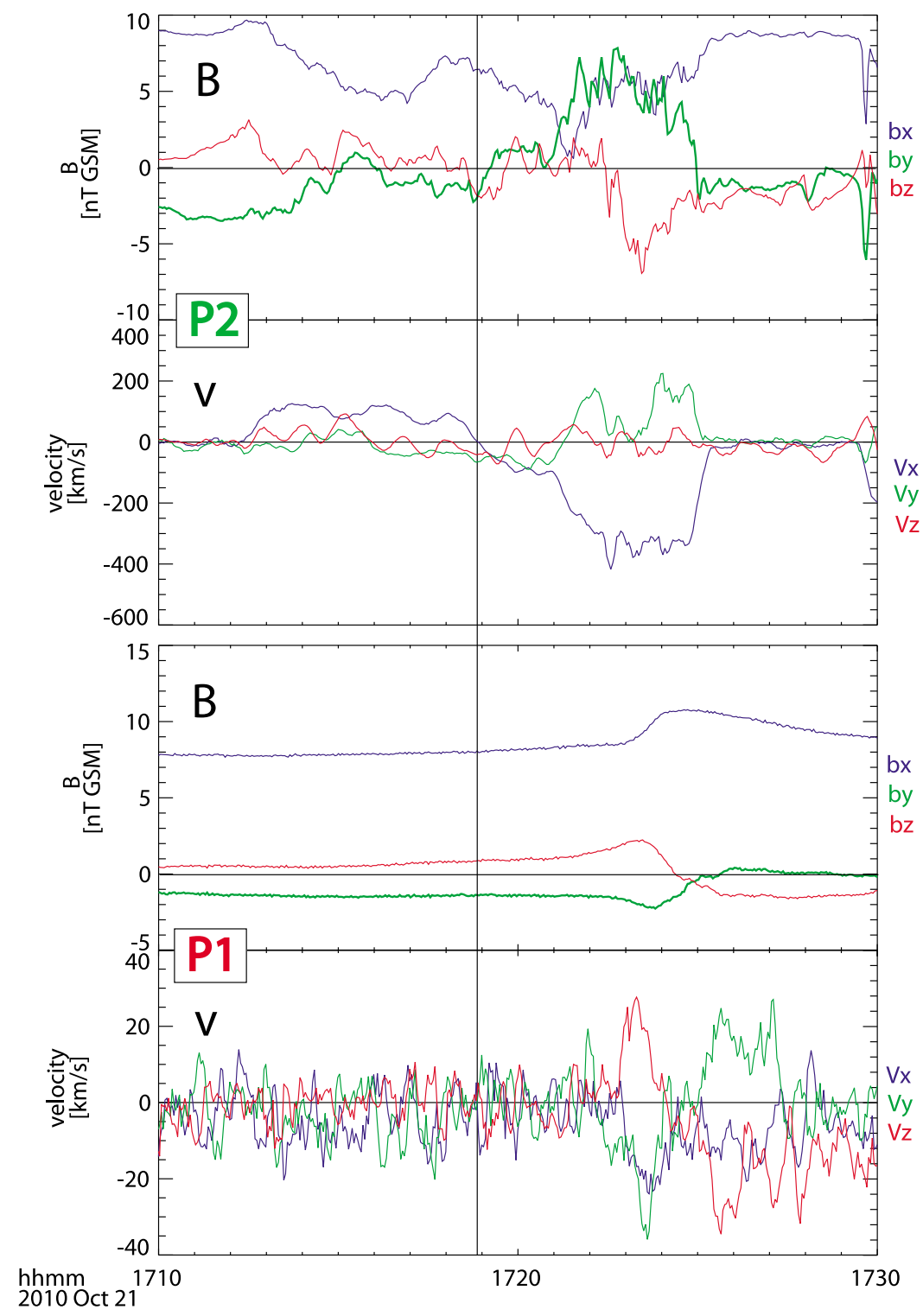

Figure 4. Magnetic field and plasma flow observations of (top) P2 and (bottom) P1 during Event 1. The solid vertical line depicts the time of the flow reversal.

tailward flow between 17:19 UT and 17:21 UT (see Figure 5). The trajectory of $\mathrm{P} 2$ during the third interval from Figure 5 is represented by a purple horizontal bar in Figure 6c. During this time P2 observed a bipolar variation in $B_{z}$, fast tailward flows (predominantly normal to the magnetic field), strong $B_{y}$, an enhancement in the total pressure and a density increase. These observations confirm the passage of the center of the flux rope (bipolar $B_{z}$ and strong $B_{y}$ ), accompanied by fast reconnection jets. At some point the structure (in a two dimensional projection it corresponds to a "plasmoid" or "O-line"), gets detached from the NENL when the last closed field line gets reconnected and clears the way for the subsequent series of flux ropes, discussed in the following section. About three minutes after P2 had observed the flow reversal, P1 detected a TCR, with a total magnetic field strength peak of 10.8 nT at $\sim 17: 24: 30 \mathrm{UT}$, yielding a compression $\Delta B / B$ of $35 \%$ with respect to the $8 \mathrm{nT}$ background. The north-then-south change in $B_{z}$ clearly indicates a tailward traveling TCR. P1 observed plasma flow of about 50 $\mathrm{km} / \mathrm{s}$ in plus (minus) $z_{G S M}$ correlated with the positive (negative) excursion of $B_{z}$. This correlation of $v_{z}$ and $B_{z}$ can be interpreted as a lifting (sinking) of plasma around the leading (trailing) part of the TCR.

[7] In general, the flow reversal observed by $\mathrm{P} 2$ can be due to either an Earthward moving X-line or a tailward moving O-line. However, the simultaneous observations of P1 allow us to rule out an Earthward moving X-line scenario. In the case of an Earthward moving X-line, one would expect to see an Earthward moving TCR observed by P1 if for an X-line that evolved tailward of $\mathrm{P} 1$ or a tailward moving TCR if for the case that the X-line evolved in between P1 and P2. These observations should occur simultaneously with the Earthward propagating flow observed by P2. Instead, P1 observed a tailward moving TCR after the flow reversal seen by P2. Hence, we associate the tailward moving TCR seen by P1 with the tailward directed flow seen by P2. 


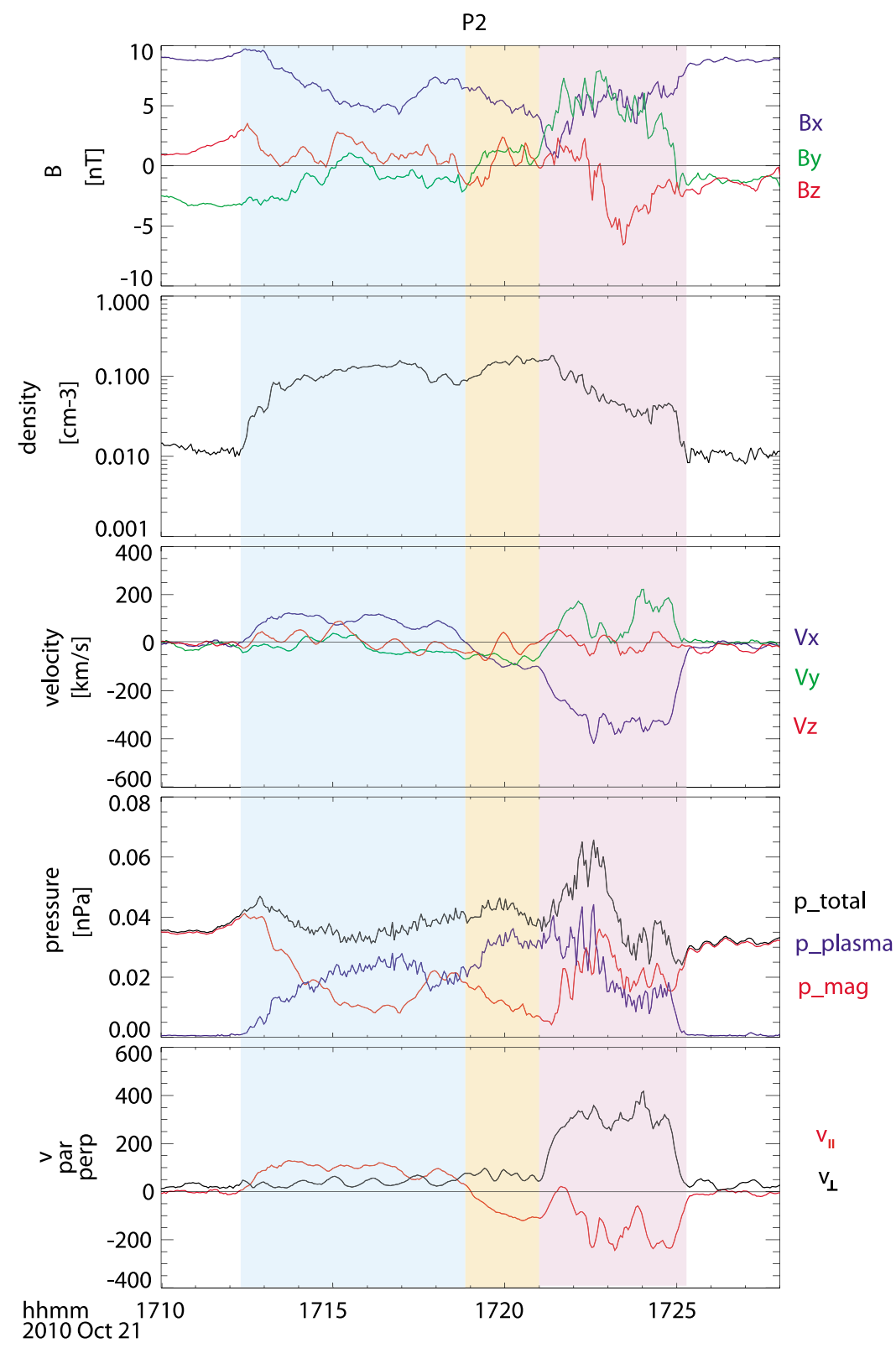

Figure 5. Observations of $\mathrm{P} 2$ during Event 1 . From top to bottom are shown magnetic field, ion density, ion velocity, pressure and parallel and perpendicular components of the plasma flow, respectively. During the first interval (blue vertical bar), P2 observed a decrease in $B_{x}$, mainly positive $B_{z}$, enhanced density, and slow Earthward flow with a strong parallel component. During the second interval (yellow vertical bar), it observed mainly positive $B_{z}$, slow tailward flow with both, parallel and perpendicular components. The third interval (purple vertical bar) is characterized by a north-then-south reversal in $B_{z}$, strong $B_{y}$, fast tailward, mainly perpendicular flow, and enhanced total pressure. An interpretation of this sequence is shown in Figure 6.

[8] The average flow speed during the Earthward (tailward) flow interval was $\sim 120(350) \mathrm{km} / \mathrm{s}$. With oppositely directed flows from a single X-line one would expect similar flow speeds in both directions. Since the tailward flow speed is significantly higher than the Earthward flow speed, we attribute the two flows to different sources. Since the lobe magnetic field strength decreased with downtail distance [e.g., Slavin et al., 1985], the lobe Alfvén velocity decreased with increasing downtail distance. Hence, we expect lower outflow speed related to a distant X-line than to a near-Earth neutral line. Consequently, it is reasonable that P2 first observed plasma outflow from the distant X-line, then tailward flow originating form a near-Earth neutral line, implying the appearance of an O-line, as depicted in Figure 6.

\section{Series of Flux Ropes/TCRs}

[9] The disturbances seen by P2 at $\sim 17: 30$ UT, 17:35:30 UT and 17:43 UT respectively (Events 2, 3 and 5), are consistent with observations of flux ropes (see Figure 7). $B_{x}$ was 
(a) 17:12 UT

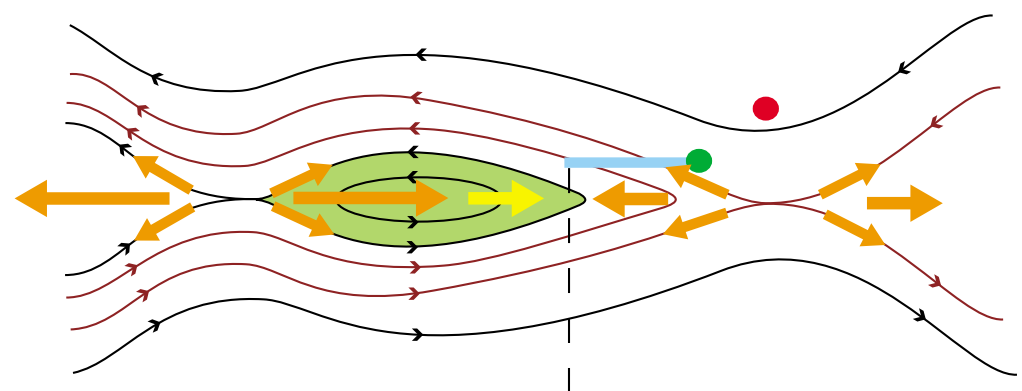

(b) 17:19 UT
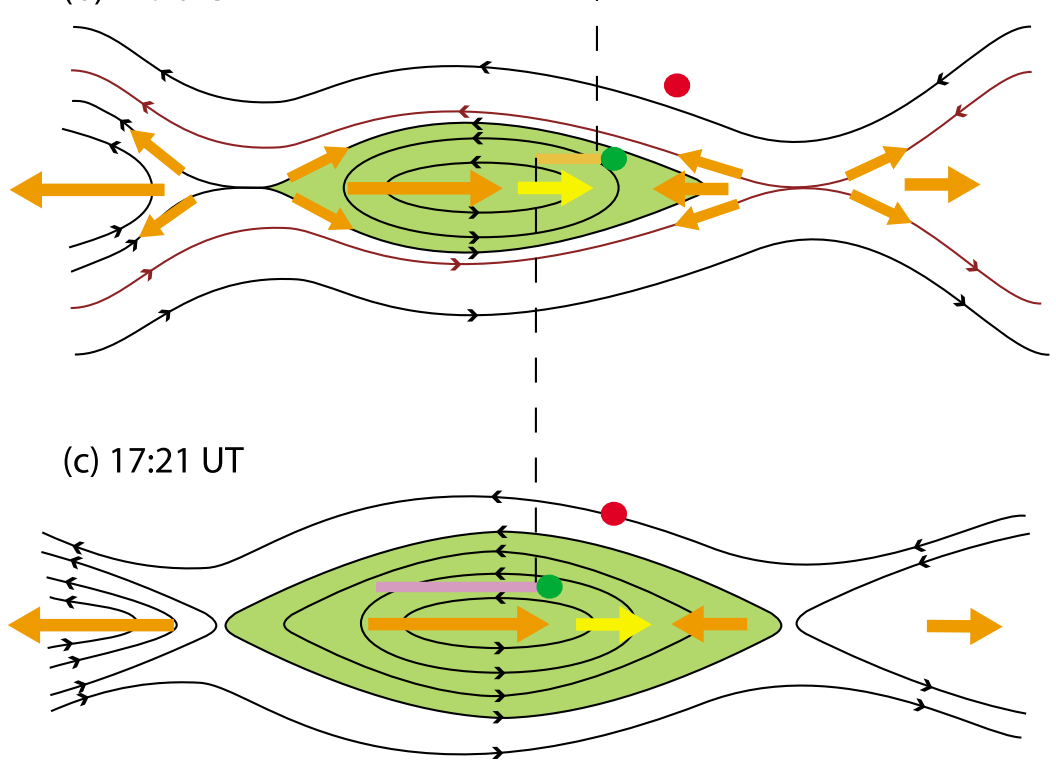

Figure 6. Schematic representation of the first event. Figures $6 a, 6 b$, and $6 c$ represent the start of the three time intervals labeled with colored vertical bars in Figure 5. (a) During the first interval, represented by a blue horizontal bar (corresponding to the first interval in Figure 5), P2 (green) observed positive $B_{z}$ and Earthward, mainly parallel flow. (b) During the second interval, denoted by an orange horizontal bar (corresponding to the second interval in Figure 5) P2 observed mainly positive $B_{z}$ and slow, tailward flow (yellow arrow). This plasma flow is interpreted as stagnant plasma that gets pushed forward by tailward reconnection outflow from a near-Earth neutral line (NENL). (c) During the third interval, denoted by a purple horizontal bar (corresponding to the third interval in Figure 5) P2 observed a bipolar variation in $B_{z}$ and fast tailward flow, accompanied by enhanced total pressure and $B_{y}$. These signatures are typical of a passing flux rope. In a two-dimensional picture this can be interpreted as the passage of an O-line, embedding both, tailward and Earthward plasma flows from the distant and near-Earth neutral line (orange arrows), respectively. P1 (red) remained outside of the structure and observed a TCR.

decreasing from its $9 \mathrm{nT}$ background, but remained positive at about $4 \sim 6.5 \mathrm{nT}$. $B_{y}$ increased from $-1 \mathrm{nT}$ to $-4.5 \sim-6 \mathrm{nT}$. Since $B_{x}$ remained at a fairly positive level and $B_{y}$ did not increase to levels comparable to the total background, P2 did not pass through the center of the flux rope. The bipolar signature in $B_{z}$ during all three events indicates a rotation of the magnetic field vector, consistent with a twisted magnetic field line structure inside flux ropes around its core axis [cf. Slavin et al., 2003; Möstl et al., 2009]. The inflection point of $B_{z}$ is around the center of the dip in $B_{x}$, as expected. All three structures were accompanied by enhanced particle fluxes, temperature, plasma pressure and tailward flows peaking between $\sim 200$ and $400 \mathrm{~km} / \mathrm{s}$. The observation of flows associated with flux rope structures is consistent with the picture of magnetic reconnection as a generation mechanism of accelerated plasma flow and magnetic field reconfiguration.

[10] At around 17:40 UT at P2, between events 3 and 5, a bipolar signature associated with a peak in the total magnetic field strength and $B_{x}$ was observed (Event 4). This signature of a TCR is confirmed by the absence of significant particle fluxes. Hence, P2 was not engulfed by the flux rope or plasma flow region during this event.

[11] After the observations made by P2, P1 detected a series of TCRs (Figure 8), identified by the typical bipolar $B_{z}$ variations and enhancements in the total magnetic field strength and $B_{x}$ at 17:31:20, 17:37, 17:41:15 and 17:44, respectively (Events 2 to 5). Although no significant particle fluxes were detected, typical variations in $v_{z}$ were observed. 


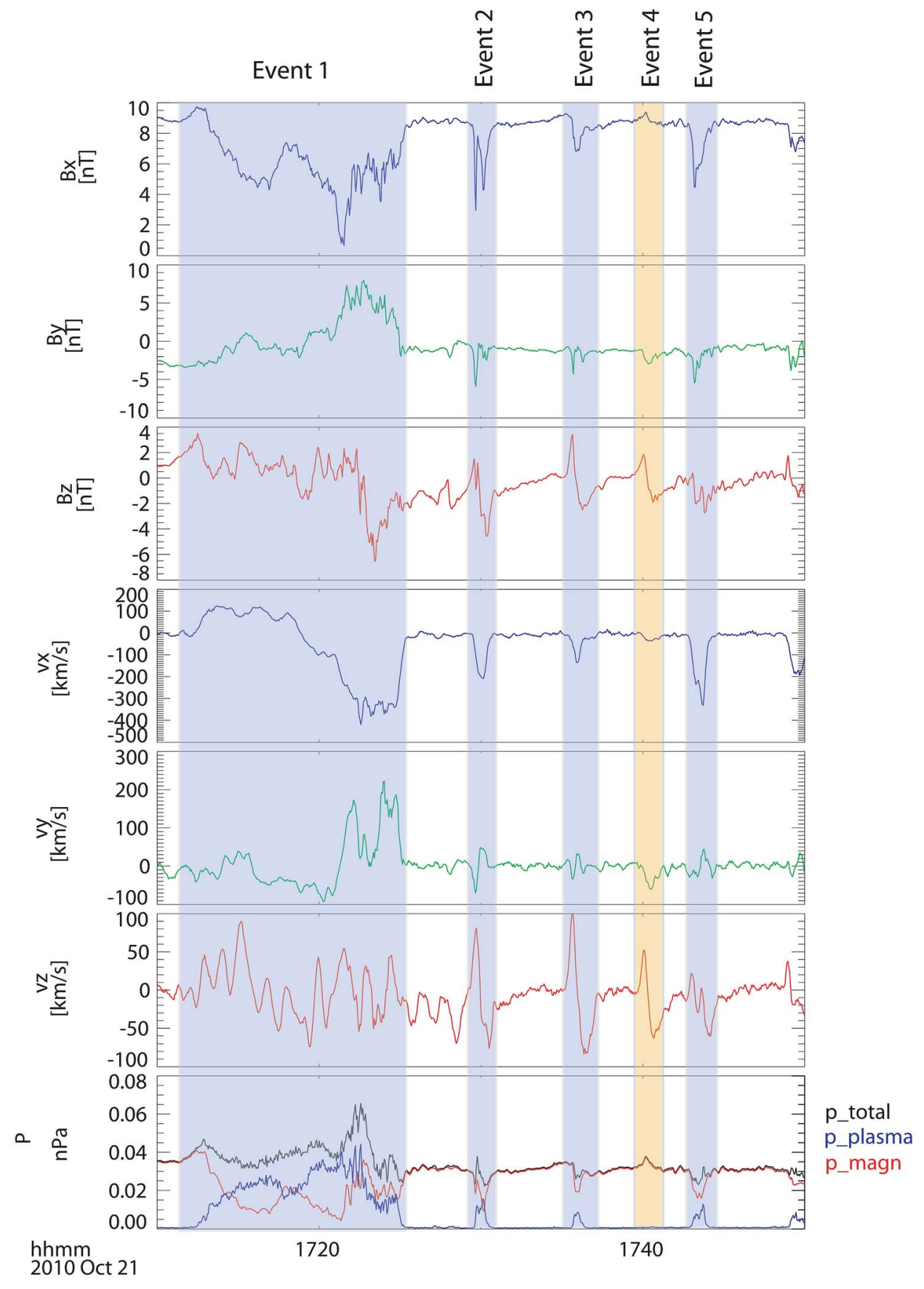

Figure 7. Observations made by $\mathrm{P} 2$ showing the five events discussed in the text. At around 17:40 a TCR is observed. Shown from top to bottom are magnetic field components $B_{x}, B_{y}, B_{z}$, plasma flow velocity $v_{x}$, $v_{y}, v_{z}$ and pressure.

[12] Due to the small spatial and temporal separation of the flux rope observations by $\mathrm{P} 2$ and TCRs by P1, we interpret the series of TCRs observed by $\mathrm{P} 2$ as remote sensing of the same flux ropes that were observed earlier by $\mathrm{P} 1$.

[13] To determine the propagation speed of the observed structures, we apply timing to three different parameters: the total pressure $P$ and components of the magnetic field $B_{x}$ and $B_{z}$. We determine the time delay between the peak in the total pressure and $B_{x}$ - related to the center of the TCR - detected by P1 with the center of the enhanced total pressure and $B_{x}$ on P2. Additionally, we determine the time delay between the inflection points in $B_{z}$ observed by $\mathrm{P} 2$ and $\mathrm{P} 1$, respectively. All three parameters $\left(P, B_{x}, B_{z}\right)$ give similar timing and hence, a similar propagation speed of the structure. Two features can be seen from Figure 9. Blue triangles, red squares and black diamonds indicate the timing results by using signatures in $B_{x}, B_{z}$ and $P$, respectively. The first structure (Event 1) propagates with the smallest propagation 


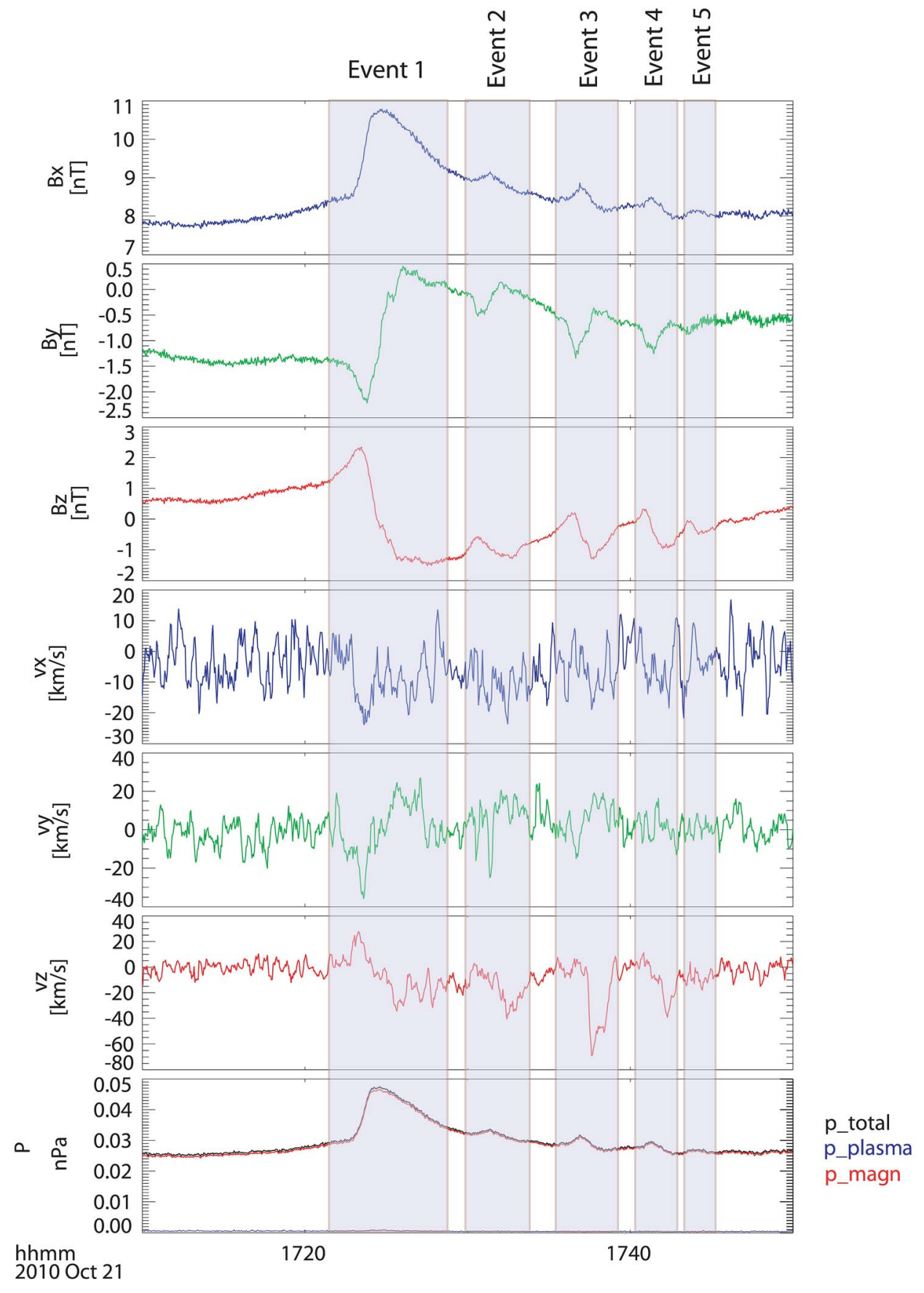

Figure 8. The five TCRs as observed by P1. Shown from top to bottom are magnetic field components $B_{x}$, $B_{y}, B_{z}$, plasma flow velocity $v_{x}, v_{y}, v_{z}$ and pressure.

speed, whereas the last structure (Event 5) shows the highest propagation speed. The velocity of the structures increases with the time of their appearance. Secondly, the propagation speeds of up to $2000 \mathrm{~km} / \mathrm{s}$ are significantly higher than the propagation velocity expected at these downtail distances $\left(-680 \pm 270 \mathrm{~km} / \mathrm{s}\right.$ at $-50>X_{G S M} \geq-100$ according to Ieda et al. [1998]). This leads to the assumption that the structures are tilted in the $x-y$-plane, exhibiting a significant $y$-component. It is also supported by a strong plasma flow component $v_{y}$ during the first event, but not during the subsequent observations.

\section{Tilted Flux Ropes}

[14] We apply a minimum variance analysis (MVA) to determine the tilt of the flux ropes observed by P2. Since the signatures of Event 1 are not very clear and Event 4 resembles a TCR, we restrict our investigations to Events 2, 3 and 5. First, we apply the MVA over the entire flux rope. The 


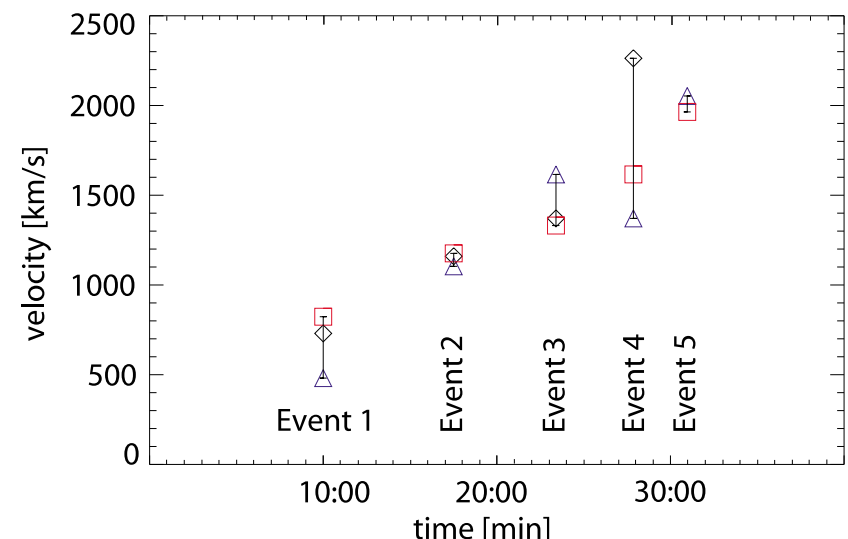

Figure 9. Propagation velocity of the five structures observed by $\mathrm{P} 2$ and $\mathrm{P} 1$ inferred from timing. Blue triangles, red squares and black diamonds indicate the timing results by using signatures in $B_{x}, B_{z}$ and $P$, respectively. The x-axis denotes the time starting at 17:12:30 UT in order to display the relative observation time in between the events, based on the inflection in $B_{z}$ at $\mathrm{P} 2$.

intermediate variance direction is expected to correspond to the principal axis direction under idealized assumptions [Slavin et al., 2003; Xiao et al., 2004]. As pointed out by Moldwin and Hughes [1991], MVA is a poor method of determining the principal axis of a flux rope, especially if the spacecraft is not passing through the center of the flux rope. Hence, we use an additional approach and compare the results. The cross-product of a flux rope's boundary normals represent its axis direction, since the boundary normals are ideally perpendicular to the axis. By applying an MVA over the leading and trailing edges of the flux rope, respectively, we determine two boundary normals during the crossing of the flux ropes boundaries. The minimum variance direction corresponds to the normal direction of the boundary separating the flux rope from its environment. We then determine the axis of the flux rope by calculating the cross product of the two boundary normals. This method implies large errors for the situation of nearly antiparallel normal directions,

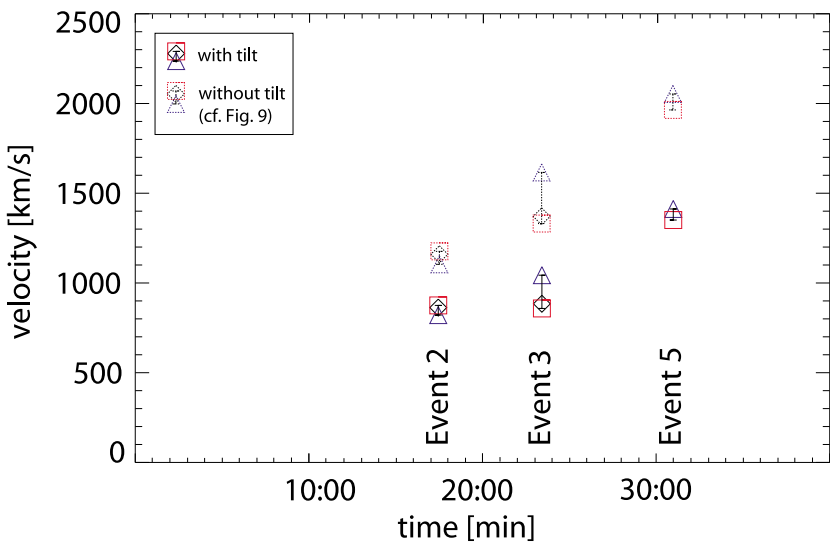

Figure 11. Same as Figure 9, but including a tilt of the structures in the $x-y-p l a n e$ as found from a principal axis determination. The propagation speed is decreased, but a trend from smaller to higher propagation speeds from the first to the last event remains. For comparison the results from the timing analysis without tilt (cf. Figure 9) are shown as dotted signs.

which is the case for crossings near the center of the flux rope. Nevertheless, both methods produce comparable results, as seen in Figure 10. The blue lines, corresponding to the axis direction in the $x-y$-plane determined via the cross product of the boundary normals, yield an axis orientation comparable to the results achieved by applying an MVA over the entire flux rope interval (black lines). The orientation of the three flux ropes is found to be $45^{\circ}, 41^{\circ}$, and $44^{\circ}$ in the $x-y-p l a n e$, respectively. This results in a reduction of the propagation speed of about 25\% (Event 2), 34\% (Event 3), 31\% (Event 5) to more reasonable average values of $870 \mathrm{~km} / \mathrm{s}$ (Event 2), $940 \mathrm{~km} / \mathrm{s}$ (Event 3), $1350 \mathrm{~km} / \mathrm{s}$ (Event 5) (see Figure 11).

\section{Correlation of $B$ and $v$}

[15] Inside the flux ropes we find a clear correlation between the magnetic field components and the corresponding plasma flow components. This indicates an internal structure with a frozen-in plasma moving together with the magnetic field. With the exception of the correlation of $v_{y}$ and

XY-GSM plane 2010-10-21/17:00:00
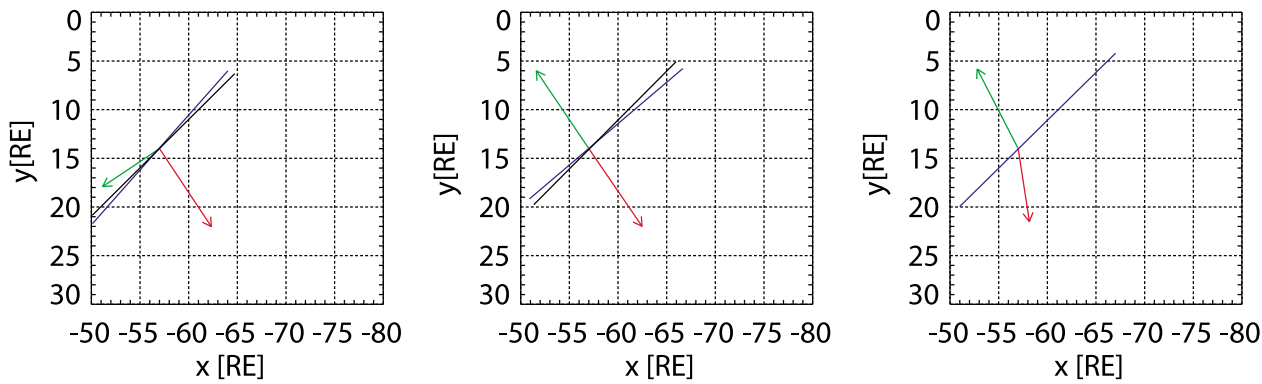

Figure 10. Axis orientation of the three flux ropes observed by P2: Events (left) 2, (middle) 3 and (right) 5 . Red: normal vector of the leading flux rope boundary, green: normal direction of the trailing boundary, blue: axis orientation as inferred from the cross product of the leading and trailing boundaries, black: axis orientation as inferred from an MVA. For FR3 the difference in the inferred axis orientation from using the two methods is too small to be displayed $\left(2^{\circ}\right)$. 

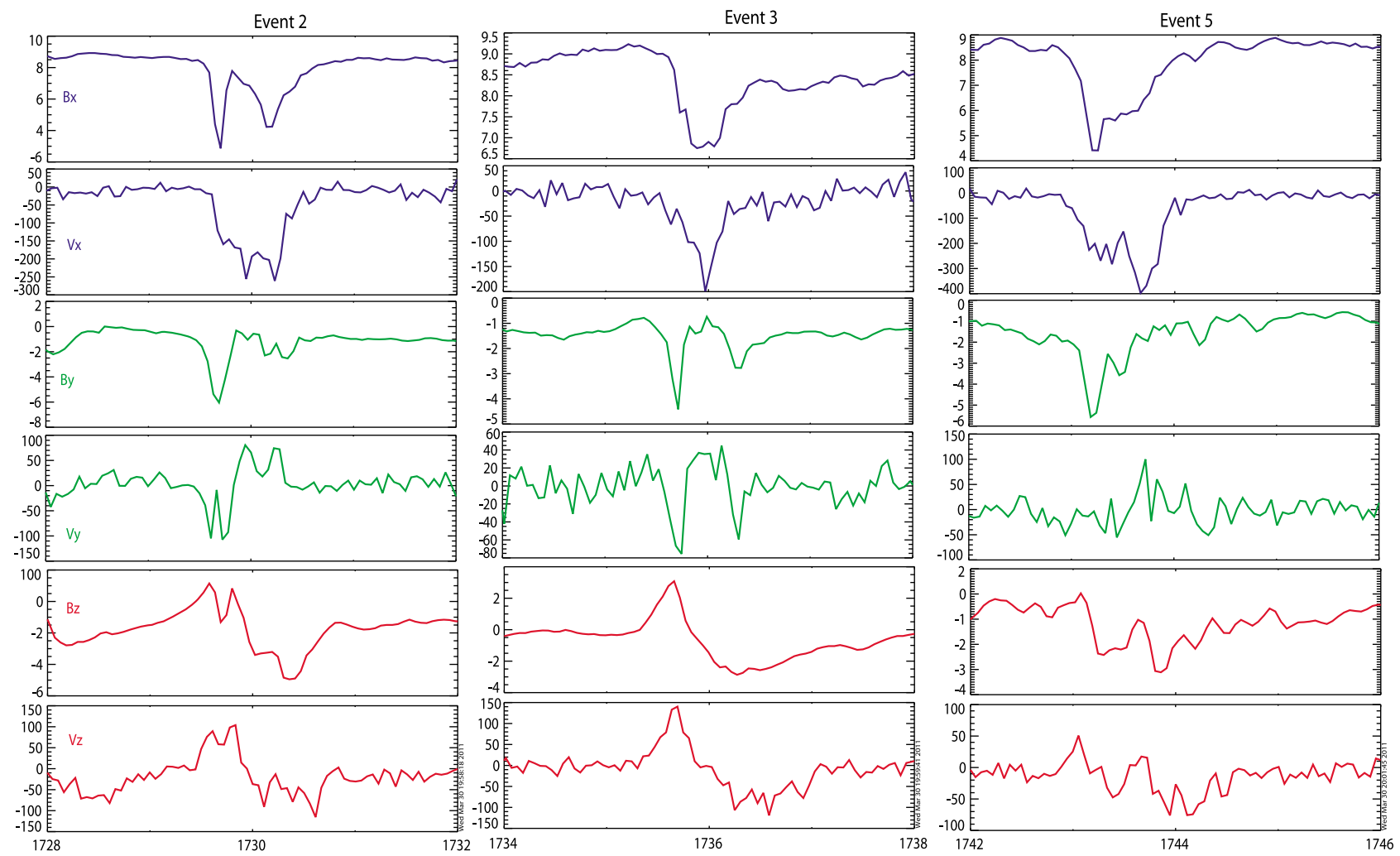

Figure 12. Magnetic field and plasma flow velocity components for the three flux ropes observed by P2. A clear correlation between the magnetic field and plasma flow components can be seen.

$B_{y}$ during Event 5, we get correlation coefficients between 0.6 and 0.95 (see Figures 12 and 13).

\section{Summary and Conclusions}

[16] ARTEMIS spacecraft P2 observed a tailward propagating O-line, followed by a series of flux ropes. Shortly after the observations at P2, P1 observed a series of TCRs associated with the flux ropes detected by $\mathrm{P} 1$. We calculated the propagation speed of the flux ropes by determining the time delay of the signatures detected on both spacecraft. The unexpectedly high propagation speeds for Events 1 to 5 between $500 \sim 2000 \mathrm{~km} / \mathrm{s}$ lead to the assumption that the structures are tilted in the $\mathrm{x}-\mathrm{y}$-plane, which is confirmed by determining the axis orientation using a minimum variance analysis. The flux ropes are tilted toward the tail by about 45 degree on the dawnside. Even when the tilt is considered, the first structure (Event 2) still exhibits the smallest propagation speed and the last one (Event 5) the highest. This gradual increase in the propagation speed can be understood if impulsive reconnection starting at the neutral sheet and progressively moving toward the boundary and eventually the lobes is the generation mechanism of these structures. Since the Alfvén speed increases from the neutral sheet to the boundary layer even if the reconnection site were to move further downtail, the reconnection rate in this hypothesis progressively increases, resulting in higher expulsion speed, explaining the observations. This leads to a sequence of flux ropes with increasing propagation velocity. Since reconnection can cease on the dawn- or duskside of the reconnection line earlier than on the other side, a tilt in the generated flux rope can be implied [Hughes and Sibeck, 1987]. Assuming symmetry of the structures with respect to the current sheet, we can estimate their minimum and maximum scale sizes with two spacecraft observations. Since P1 does not enter the flux ropes, its distance to the nominal neutral sheet of about $4 R_{E}$ (obtained from http://sscweb.gsfc.nasa.gov/ tipsod/) depicts the maximum extent of the structures into the northern part of the tail. During Event 4, both spacecraft observe a TCR and remain outside of the flux ropes. Since P2 is located $1 R_{E}$ north of the nominal neutral sheet, the fourth structure corresponds to a particularly small flux rope. In GSM-y the spacecraft are separated by about $3 R_{E}$. Since both spacecraft observe flux rope signatures, their separation gives the minimum width of the structures in the GSM-ydirection. From the periods of enhanced plasma pressure on P2 we can determine the duration of the flux rope encounters. For the three ropes we get 56, 48 and $70 \mathrm{~s}$, respectively. With the propagation velocity inferred from inter-spacecraft timing, taking into account the tilted axis of the structure, the size of the flux ropes along the propagation path is $7.6,7$ and $14.8 R_{E}$ for Events 2, 3 and 5, respectively. Thus, the last structure extends over the spacecraft separation, which is obvious in the observed data since P1 detected the TCR at a the same time as P2 encountered the flux rope. The observed flux ropes near lunar orbit exhibit small scale length normal to the current sheet of only 2 to $8 R_{E}$ in $z_{G S M}$ and are significantly inclined with respect to the noon-midnight meridian. We propose two scenarios for the creation of tilted flux ropes. 

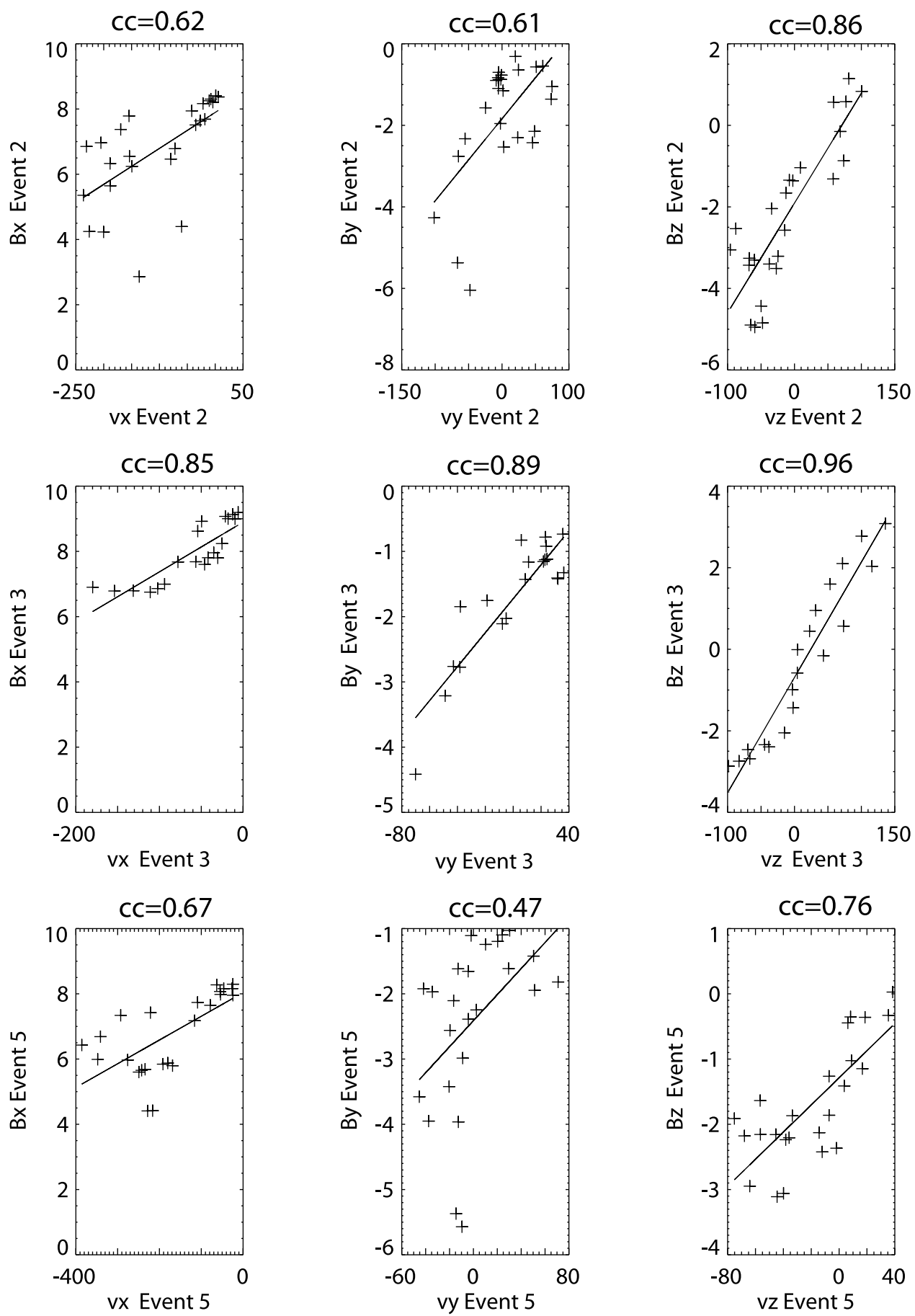

Figure 13. Correlation coefficients for magnetic field and plasma flow components from Figure 12.

Both scenarios imply the presence of $B_{y}$ in the plasma sheet and are sketched in Figure 14.

[17] 1. Reconnection starts near midnight and progressively spreads into the dusk- and dawn-sectors. In other words, the reconnection line gradually extends over the $y$-direction and reconnection appears differential. Reconnection of field lines near midnight leads to the formation of a flux rope over the extent of the so-far present reconnection line. This first and central part of the flux rope moves tailward with the accelerated plasma but does not get fully released since its ends are still connected to the ionosphere. Simultaneously, the reconnection line spreads farther into the dusk- and dawn-sectors and adds more twists to the already established flux rope. Since the previously formed flux rope has been transported a certain distance downtail already, this newly formed part of the flux rope is trailing the previously formed central part. This leads to a boomerang-shaped flux rope, exhibiting a non-uniform shape with inclined "wings". In this scenario, the determined flux rope axis direction from observations by $\mathrm{P} 2$ corresponds only to a local axis direction. 

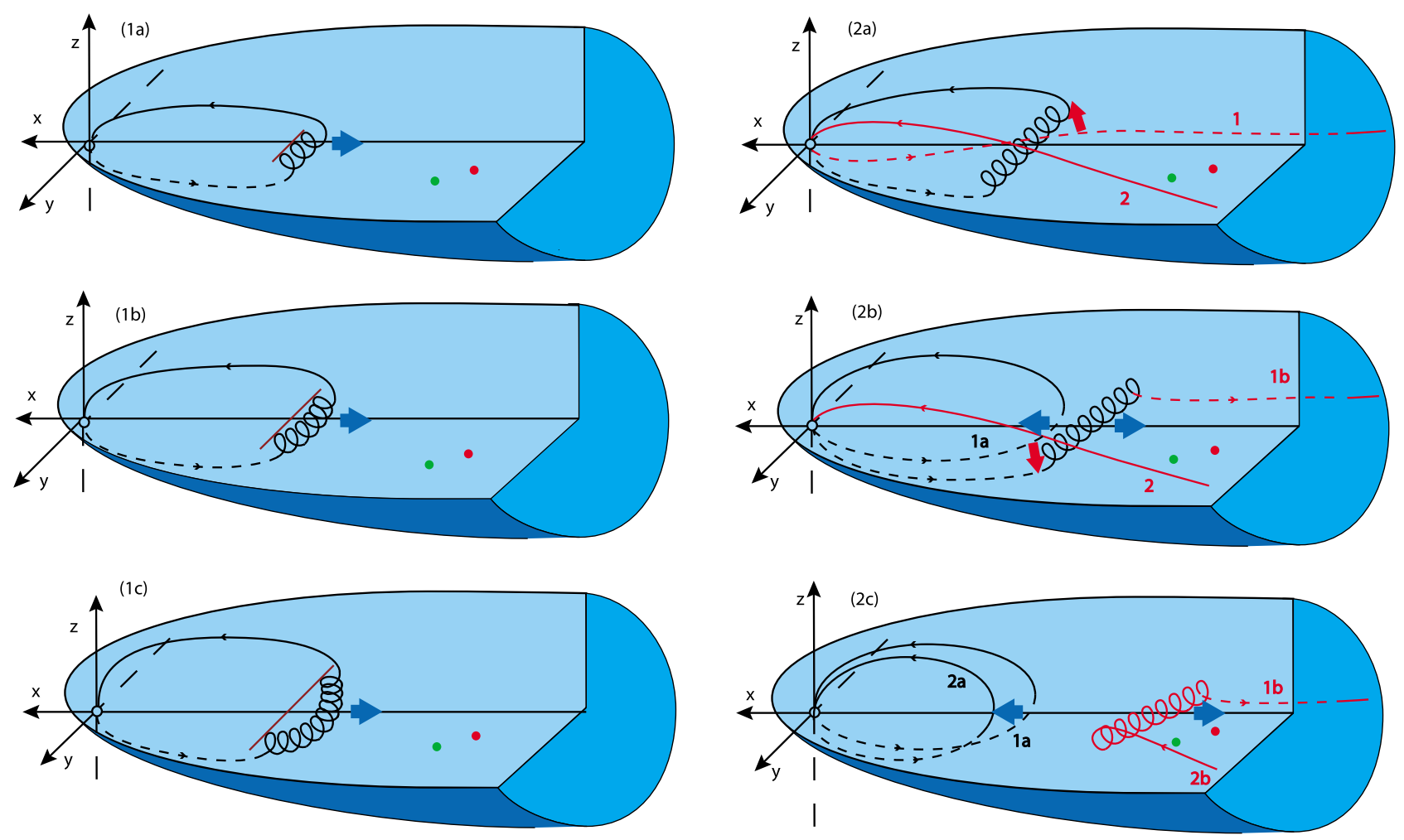

Figure 14. Two possible scenarios for the formation of tilted flux ropes in the Earth's magnetosphere (blue). In the initial state of this idealized sketch, the flux rope is resting within the equatorial plane, exhibiting a dawn-dusk extent and is connected at its ends to the Earth's ionosphere. The two spacecraft P1 and P2 are symbolized by red and green dots, respectively. (left) Reconnection starts near midnight and forms the central segment of the flux rope (step 1a). The reconnection line is indicated by a brown line. As an assumption, the reconnection line spreads along the east-west direction and adds additional twists to the flux rope. Since the central part (formed during step 1a) has moved farther downtail, the newly added twists are trailing the central part (step 1b). As the reconnection line spreads farther along the east-west direction, the flux rope establishes a non-uniform, boomerang-like shape. This also implies that the flux rope is thicker at its center than at its flanks. For simplicity, only one flux rope field line is drawn. (right) Open Field line 1 (located in the southern lobe in the dawn sector) reconnects on the dawnside end of the flux rope (step 2a). Now, this end of the flux rope is not connected to the ionosphere any more and moves into the tail. This leads to a tilting of the flux rope, since the duskside end is still connected to the ionosphere until open field line 2 (located in the northern lobe in the dusk sector) reconnects at this end (step 2b). With no connection to the ionosphere, the flux rope now propagates into the tail (step 2c).

Due to the non-uniform boomerang-like shape, the flux rope does not exhibit a uniform axis direction across its entire extent. If reconnection at the central portion of the connection line continues as its extent in the east-west-direction increases, this scenario also implies that the flux rope is thicker at its center than at its flanks.

[18] 2. In order to disconnect the flux rope from the ionosphere, field lines at its ends need to reconnect with open field lines [Hughes and Sibeck, 1987]. This must not necessarily happen at both ends at the same time. While one end (in Figure 14 (step 2b) the dawnside end) is already reconnected to an open field line, the other end is still connected to the ionosphere. Hence, the dawnside end will be pulled downtail by the open field line, while the duskside end is still connected to the Earth. This differential motion leads to a tilt in the flux rope. This non-simultaneous reconnection of open field lines at either end of the flux rope can be due to either a differential reconnection rate along the reconnection line or due to asymmetries in the non-ideal field line configuration in the magnetotail. When the other end of the flux rope is finally reconnected to an open field line as well, the flux rope is no longer connected to the ionosphere and is free to move downtail, exhibiting a tilt due to the non-simultaneous reconnection of the flux ropes' ends with open field lines.

[19] Certainly, we do not rule out a combination of both scenarios, i.e., a progressive spreading of the reconnection line in $y$, leading to a non-uniform flux rope (scenario 1) followed by a tilting due to non-simultaneous reconnection of open field lines at its ends (scenario 2). We do not see a physical reason for either constant reconnection in the equatorial plane or for simultaneous reconnection of open field lines at both ends of the flux rope under realistic conditions. Hence, the appearance of tilted flux ropes can be a common phenomenon as observed in previous studies [Slavin et al., 2003; Walsh et al., 2007]. This tilt influences the determination of propagation speeds of flux ropes and 
studies based on the arrival times can be biased if a possible tilt of the structures is not taken into account.

[20] Acknowledgments. This work was supported by NASA grant NAS5-0299 and German Ministry for Economy and Technology, the German Center for Aviation and Space (DLR), contract 50QP0402, by a Marie Curie International Outgoing Fellowship within the 7th European Community Framework Programme and the Austrian Science Fund (FWF): J3041-N16. We acknowledge C. W. Carlson and J. P. McFadden for use of ESA data and D. Larson and R. P. Lin for use of SST data. We thank D. Sibeck, M. Kivelson and K. Khurana for useful discussions.

[21] Masaki Fujimoto thanks the reviewers for their assistance in evaluating this paper.

\section{References}

Angelopoulos, V. (2008), The THEMIS mission, Space Sci. Rev., 141, 5-34. Angelopoulos, V. (2011), The ARTEMIS mission, Space Sci. Rev., 165, 3-25, doi:10.1007/s11214-010-9687-2.

Auster, H. U., et al. (2008), The THEMIS fluxgate magnetometer, Space Sci. Rev., 141, 235-264.

Baker, D., R. Anderson, R. Zwickl, and J. Slavin (1987), Average plasma and magnetic field variations in the distant magnetotail associated with near-earth substorm effects, J. Geophys. Res., 92(A1), 71-81.

Hones, E. W., Jr. (1976), The magnetotail: Its generation, and dissipation, in Physics of Solar Planetary Environments, vol. II, edited by D. J. Williams, pp. 558-571, AGU, Washington, D. C.

Hones, E. W., Jr. (1977), Substorm processes in the magnetotail: Comments on "On hot tenuous plasmas, fireballs, and boundary sayers in the Earth's magnetotail" by L. A. Frank, K. L. Ackerson, and R. P. Lepping, J. Geophys. Res., 82(35), 5633-5640.

Hones, E. W., Jr., J. Birn, D. N. Baker, S. J. Bame, W. C. Feldman, D. J McComas, R. D. Zwickl, J. A. Slavin, E. J. Smith, and B. T. Tsurutan (1984), Detailed examination of a plasmoid in the distant magnetotail with ISEE 3, Geophys. Res. Lett., 11(10), 1046-1049.

Hoshino, M., T. Mukai, A. Nishida, Y. Saito, T. Yamamoto, and S. Kokubun (1996), Evidence of two active reconnection sites in the distant magnetotail, J. Geomagn. Geoelectr., 48, 515-523.

Hughes, W. J., and D. G. Sibeck (1987), On the 3-dimensional structure of plasmoids, Geophys. Res. Lett., 14(6), 636-639, doi:10.1029/ GL014i006p00636.

Ieda, A., S. Machida, T. Mukai, Y. Saito, T. Yamamoto, A. Nishida, T. Terasawa, and S. Kokubun (1998), Statistical analysis of the plasmoid evolution with Geotail observations, J. Geophys. Res., 103(A3), 4453-4465.

Ieda, A., D. Fairfield, T. Mukai, Y. Saito, S. Kokubun, K. Liou, C.-I. Meng G. Parks, and M. Brittnacher (2001), Plasmoid ejection and auroral brightenings, J. Geophys. Res., 106(A3), 3845-3857.

Khurana, K. K., M. G. Kivelson, L. A. Frank, and W. R. Paterson (1995), Observations of magnetic flux ropes and associated currents in Earth's magnetotail with the Galileo spacecraft, Geophys. Res. Lett., 22(16), 2087-2090

Kivelson, M., et al. (1993), The Galileo Earth encounter: Magnetometer and allied measurements, J. Geophys. Res., 98(A7), 11,299-11,318.

Machida, S., A. Ieda, T. Mukai, Y. Saito, and A. Nishida (2000), Statistical visualization of Earth's magnetotail during substorms by means of multidimensional superposed epoch analysis with Geotail data, J. Geophys. Res., 105(A11), 25,291-25,303.

McFadden, J. P., C. W. Carlson, D. Larson, V. Angelopolos, M. Ludlam, R. Abiad, and B. Elliot (2008), The THEMIS ESA plasma instrument and inflight calibration, Space Sci. Rev., 141, 277-302.

Moldwin, M. B., and W. J. Hughes (1991), Plasmoids as magnetic flux ropes, J. Geophys. Res., 96(A8), 14,051-14,064, doi:10.1029/ 91JA01167.

Moldwin, M. B., and W. J. Hughes (1992a), On the formation and evolution of plasmoids: A survey of ISEE 3 Geotail data, J. Geophys. Res., 97(A12), 19,259-19,282.

Moldwin, M. B., and W. J. Hughes (1992b), Multi-satellite observations of plasmoids: IMP 8 and ISEE 3, Geophys. Res. Lett., 19(11), 1081-1084.
Möstl, C., C. F. Farrugia, H. K. Biernat, S. A. Kiehas, R. Nakamura, V. V. Ivanova, and Y. Khotyaintsev (2009), The structure of an earthward propagating magnetic flux rope early in its evolution: Comparison of methods, Ann. Geophys., 27, 2215-2224, doi:10.5194/angeo-27-2215-2009.

Mukai, T., et al. (1996), Structure and kinetic properties of plasmoids and their boundary regions, J. Geomagn. Geolectr., 48, 541-560.

Mukai, T., et al. (1998), Dynamics and kinetic properties of plasmoids and flux ropes: Geotail observations, in New Perspectives on the Earth's Magnetotail, Geophys. Monogr. Ser., vol. 105, edited by A. Nishida, D. N. Baker, and S. W. H. Cowley, pp. 117-132, AGU, Washington, D. C.

Nagai, T., K. Takahashi, H. Kawano, T. Yamamoto, S. Kokubun, and A. Nishida (1994), Initial GEOTAIL survey of magnetic substorm signatures in the magnetotail, Geophys. Res. Lett., 21(25), 2991-2994.

Nagai, T., M. Fujimoto, M. Nakamura, R. Nakamura, Y. Saito, T. Mukai, T. Yamamoto, A. Nishida, and S. Kokubun (1998), A large southward magnetic field of $-23.5 \mathrm{nT}$ in the January 10, 1995, plasmoid, J. Geophys. Res., 103(A3), 4441-4451.

Nishida, A., T. Mukai, Y. Saito,T. Yamamoto, H. Hayakawa, K. Maezawa, S. Machida, T. Terasawa, S. Kokubun, and T. Nagai (1994), Transition from slow flow to fast tailward flow in the distant plasma sheet, Geophys. Res. Lett.,21, 2939-2942.

Richardson, I., S. Cowley, E. Hones Jr., and S. Bame (1987), Plasmoidassociated energetic ion bursts in the deep geomagnetic tail: Properties of plasmoids and the postplasmoid plasma sheet, J. Geophys. Res., 92(A9), 9997-10,013.

Schindler, K. (1974), A theory of substorm mechanism, J. Geophys. Res., 79(19), 2803-2810, doi:10.1029/JA079i019p02803.

Sibeck, D. G., et al. (2011), ARTEMIS science objectives and mission phases, Space Sci. Rev., 165, 59-91, doi:10.1007/s11214-011-9777-9.

Slavin, J. A., E. J. Smith, B. T. Tsurutani, D. G. Sibeck, H. J. Singer, D. N. Baker, J. T. Gosling, E. W. Hones, and F. L. Scarf (1984), Substorm associated traveling compression regions in the distant tail: ISEE-3 Geotail observations, Geophys. Res. Lett., 11(7), 657-660.

Slavin, J. A., E. J. Smith, D. G. Sibeck, D. N. Baker, R. D. Zwickl, and S. I. Akasofu (1985), An ISEE 3 study of average and substorm conditions in the distant magnetotail, J. Geophys. Res., 90(A11), 10,875-10,895, doi:10.1029/JA090iA11p10875.

Slavin, J. A., et al. (1989), CDAW 8 observations of plasmoid signatures in the geomagnetic tail: An assessment, J. Geophys. Res., 94(A11) $15,153-15,175$

Slavin, J. A., M. F. Smith, E. L. Mazur, D. N. Baker, E. W. Hones Jr., T. Iyemori, and E. W. Greenstadt (1993), ISEE 3 observations of traveling compression regions in the Earth's magnetotail, J. Geophys. Res., 98(A9), 15,425-15,446

Slavin, J. A., et al. (1998), ISTP observations of plasmoid ejection: IMP 8 and Geotail, J. Geophys. Res., 103(A1), 119-133.

Slavin, J. A., et al. (1999), Dual spacecraft observations of lobe magnetic field perturbations before, during and after plasmoid release, Geophys. Res. Lett., 26(19), 2897-2900.

Slavin, J. A., et al. (2002), Simultaneous observations of earthward flow bursts and plasmoid ejection during magnetospheric substorms, J. Geophys. Res., 107(A7), 1106, doi:10.1029/2000JA003501.

Slavin, J. A., et al. (2003), Geotail observations of magnetic flux ropes in the plasma sheet, J. Geophys. Res., 108(A1), 1015, doi:10.1029/ 2002JA009557.

Taguchi, S., J. A. Slavin, and R. P. Lepping (1997), IMP 8 observations of traveling compression regions in the mid-tail near substorm expansion phase onset, Geophys. Res. Lett., 24(4), 353-356.

Walsh, A. P., A. N. Fazakerley, R. J. Wilson, I. V. Alexeev, P. D. Henderson, C. J. Owen, E. Lucek, C. Carr, and I. Dandouras (2007) Near-simultaneous magnetotail flux rope observations with Cluster and Double Star, Ann. Geophys, 25, 1887-1897.

Xiao, C. J., Z. Y. Pu, Z. W. Ma, S. Y. Fu, Z. Y. Huang, and Q. G. Zong, (2004), Inferring of flux rope orientation with the minimum variance analysis technique, J. Geophys. Res., 109, A11218, doi:10.1029/ 2004JA010594. 